\title{
SECHS FRAGEN ZU OPEN ACCESS AN ... TU WIEN ACADEMIC PRESS
}

\section{von Diana Wernisch, Silvia Ziemkendorf und Andreas Ferus}

Zusammenfassung: Im Rahmen dieses Beitrags beantworten Vertreter_innen von TU Wien Academic Press sechs Fragen rund um das Thema Open Access. Der Beitrag entstand im Zuge der Tätigkeit der Unterarbeitsgruppe „Universitätsverlage und universitätsnahe Verlage" des Teilprojekts 4 „Förderung von Open Access-Publikationen und alternativen Open Access-Publikationsmodellen" des Hochschulraumstrukturmittelprojekts Austrian Transition to Open Access (AT2OA) (https://at2oa.at/ueber.html) und ist Teil einer Serie von Interviews, die - in Anlehnung an die Reihe „Fünf Fragen an ... “ der Universitätsbibliothek der Technischen Universität Berlin - initiiert wurde, um mehr über die Haltung österreichischer Verlage zum Thema Open Access zu erfahren. *

Schlagwörter: TU Wien Academic Press; Open Access; Wissenschaftsverlag

\section{SIX QUESTIONS ABOUT OPEN ACCESS TO ... TU WIEN ACADEMIC PRESS}

Abstract: In this article representatives of TU Wien Academic Press answer six questions around the topic of Open Access. The contribution was developed in the course of the work of the sub-working group "University presses and universityrelated publishers" of the sub-project 4 "Promotion of Open Access Publications and Alternative Open Access Publication Models" of the Higher Education Area Structural Funds project Austrian Transition to Open Access (AT2OA) (https:// www.at2oa.at/en/home.html) and is part of a series of interviews initiated on the basis of the series "Five questions to ..." of the Berlin University of Technology library to find out more about the attitude of Austrian publishers towards Open Access.

Keywords: TU Wien Academic Press; Open Access; scientific publisher

DOI: https://doi.org/10.31263/voebm.v72i2.3027

(c) Diana Wernisch, Silvia Ziemkendorf, Andreas Ferus

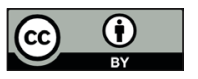

Dieses Werk ist lizenziert unter einer

Creative-Commons-Lizenz Namensnennung 4.0 International 


\section{Bitte stellen Sie uns Ihren Verlag in wenigen Sätzen kurz vor? Was ist das Selbstverständnis Ihres Verlages?}

TU Wien Academic Press ist ein wissenschaftlicher Open-Access-Verlag und wird als Service und Non-Profit-Einrichtung von der TU Wien Bibliothek betrieben. Der Verlag fördert durch professionelle Publikationsunterstützung eine offene Wissenschafts- und Publikationskultur („Open Science") im Rahmen der Forschungsschwerpunkte und strategischen Ausrichtung der TU Wien.

Im Verlag werden forschungsbasierte Buchpublikationen aus dem gesamten Fächerspektrum der TU Wien veröffentlicht. Das E-Book im OpenAccess-Zugriff ist das Standardformat des Verlags. Optional können Bücher zusätzlich als Printversion herausgegeben werden.

Als Wissenschaftsverlag ist für TU Wien Academic Press die Qualitätssicherung von besonderer Bedeutung. Verlagsseitige Maßnahmen zur Gewährleistung hoher wissenschaftlicher Qualität sind daher Standard in unserem Publikationsprogramm und die doppelte externe Begutachtung ist der Regelprozess für Publikationen in unserem Verlag. Verschiedene Unterstützungspakete des Verlags stellen Qualität in verschiedenen Dimensionen sicher (fachwissenschaftliche Qualitätssicherung, Formalaspekte, Formatstandards etc.).

\section{Was bewegt Sie dazu, ein Open-Access-Geschäftsmodell anzubieten? Vor welchen Herausforderungen stehen Sie als Verlag hinsichtlich Open Access?}

TU Wien Academic Press steht für eine offene Wissenschafts- und Publikationskultur an der TU Wien. Mit dem Verlag bietet die TU Wien Bibliothek seit 2018 nicht nur effiziente und transparente Publikationsunterstützung für die Open-Access-Veröffentlichung von Zeitschriftenartikeln und Journals, sondern auch maßgeschneiderte Lösungen für Buchpublikationen, wie sie an einer technischen Universität relevant sind. Mit dem Open-Access-E-Book als kostenfreiem Standardformat unterstützt der Verlag alle Forschenden im Sinne der Open-Access-Policy der TU Wien.

Für die Wissenschaftler_innen ist unser Open-Access-Publikationsmodell aus Gründen der maximalen Verbreitung ihrer Forschungsergebnisse attraktiv. TU Wien Academic Press ist außerdem ein interessanter Partner, weil die Autor_innen nur einfache Nutzungsrechte an den Verlag abtreten müssen und den Forschenden damit jegliche weitere Verwertungsmöglich- 
keit weiterhin offen steht. Ein Open-Access-Geschäftsmodell ist daher ein Modell, das auf die Bedürfnisse unserer Forschenden zugeschnitten ist.

Ein Open-Access-Buch mit der Zusatzoption einer Printveröffentlichung schafft beste Voraussetzungen für die größtmögliche Verbreitung einer Publikation. Dennoch ist dieser Prozess sowie das Monitoring der Rezeption bei einem Open-Access-Buch heute noch eine Herausforderung, da die Publikationssysteme und -kanäle im Vergleich zu Zeitschriftenpublikationen weniger einheitlich strukturiert sind.

\section{Welche Möglichkeiten zum Open-Access-Publizieren haben Autor_innen in Ihrem Verlag? Welche Services bieten Sie Autor_innen im Bereich Open Access?}

Die frei im Internet verfügbare Open-Access-Publikation ist das Standardformat bei TU Wien Academic Press. Dabei können die Autor_innen zwischen vier verschiedenen Creative Commons-Lizenzmodellen wählen. Zudem unterstützt ein breites, kostenloses Servicepaket für Open-Access-EBooks die Wissenschaftler_innen mit zahlreichen Leistungen:

- Design und Satz des Covers

- Unterstützung bei Struktur, Formalaspekten und Satz des Innenteils

- Formallektorat des Manuskriptes

- professionelle Veröffentlichungsformate (z. B. druck-, veröffentlichungs- und archivierungsfähige PDF-Formate)

- Beratung in Rechtsfragen, wie bspw. hinsichtlich Urheber- und Bildrechten sowie bei der Auswahl geeigneter Lizenzmodelle (CCLizenzen)

- Vermittlung und Förderung von Übersetzungen/Korrektorat/Lektorat

- Beratung zu Fördermöglichkeiten

- Unterstützung bei der Recherche nach oder der Erstellung von Bildern, Grafiken etc.

- langfristige Planung und Co-Konzeptionierung einer Buchherausgabe (z. B. bei Konferenzen)

- zeitliche Detailplanung des Buchprojektes

- Qualitätssicherung und Zusammenarbeit bei Prozessen des Peer Reviews

- professionelle Referenzierung, Indexierung und Verbreitung über verschiedenste Systeme

- Vermarktung des Buches, auch von E-Books, z. B. durch Rezensionen, Newsmeldungen, Features 
Zusätzlich zu einer Open-Access-Publikation besteht eine Print-Zusatzoption. Im Regelfall müssen die Autor_innen hier (z. B. über Drittmittelprojekte) die Druckkosten vorfinanzieren, wobei unser Geschäftsmodell Rückerstattungen aus Einnahmen ermöglicht.

\section{Wie kalkulieren Sie Open-Access-Publikationen? Können Sie uns viel- leicht ein Preis-Beispiel für eine Standardpublikation nennen?}

Open-Access-Publikationen sind bei uns grundsätzlich kostenfrei.

\section{Was wünschen Sie sich von den Universitätsbibliotheken und Förder- einrichtungen in Bezug auf die Finanzierung von Open-Access-Büchern?}

Für Fördereinrichtungen ist es wichtig, die Entwicklung am europäischen Open-Access-Verlagsmarkt zu beobachten und die dynamische Entwicklung im Bereich Universitätsverlage bzw. an den Bedürfnissen der Wissenschaftler_innen orientierter Verlage gegebenenfalls zu stärken.

Wünschenswert wäre der Abbau von Hindernissen und Unklarheiten bezüglich der Antragsstellung und Abwicklung bei den Förderinstitutionen und anderen Partnern im Zusammenhang mit der Verbreitung von OpenAccess-Büchern (z. B. Literar-Mechana, Amazon-Listungen, Scopus). Das wäre nicht nur im Sinne der Autor_innen, sondern auch der Universitätsverlage, die durch die Einbindung in die Universitätsstruktur oft als Körperschaft öffentlichen Rechts agieren.

Die Sicherstellung aussagekräftiger Informationen in Verbreitungssystemen sowie die weitere Erhöhung der Zugänglichkeit oder auch der Bezuschussung sind wichtige Punkte. Hier ist der gegenseitige Informationsaustausch unerlässlich.

\section{Gibt es aus Ihrer Sicht Publikationen, die sich für Open Access beson- ders gut bzw. kaum/überhaupt nicht eignen?}

Nein, bisher haben wir keine derartigen Erfahrungen gemacht. Allerdings ist es bei Open-Access-Publikationen, die häufig über CC-Lizenzmodelle verbreitet werden, sehr wichtig, die Herausgeber_innen und Autor_innen über die rechtliche Situation gut zu informieren. Ebenso müssen die Prozesse (z. B. bei der Herausgabe von Conference Proceedings ab der Ver- 
öffentlichung des Calls) so aufgesetzt werden, dass Wissen und Einigkeit über die gewählte Lizenzierung besteht und alle Rechte (beispielsweise bei der Verwendung urheberrechtlich geschützter Bilder) gewahrt werden.

\section{TU Wien Academic Press \\ c/o TU Wien Bibliothek \\ TU Wien \\ Resselgasse 4, 1040 Wien}

E-Mail: academicpress@tuwien.ac.at

Website: www.tuwien.at/academicpress

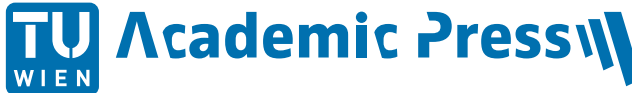

Mag. ${ }^{\text {D Dr. }}{ }^{\text {in }}$ Diana Wernisch

ORCID: https://orcid.org/0000-0002-4251-9172 Technische Universität Wien, TU Wien Academic Press

E-Mail: diana.wernisch@tuwien.ac.at

Silvia Ziemkendorf Technische Universität Wien, TU Wien Academic Press E-Mail: silvia.ziemkendorf@tuwien.ac.at

Mag. Andreas Ferus, MSc ORCID: http://orcid.org/0000-0003-2509-0009 Akademie der bildenden Künste Wien, Universitätsbibliothek E-Mail: a.ferus@akbild.ac.at

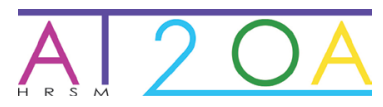


* Es sei an dieser Stelle Dagmar Schobert, Leiterin der UB der TU Berlin, für die Erlaubnis zur Übernahme des Konzepts und Christian Kaier für sein Feedback hinsichtlich der Fragen gedankt. In der erwähnten Interviewreihe der UB der TU Berlin sind im Übrigen bis dato folgende Beiträge erschienen:

- FünfFragen an ... Barbara Budrich (https://blogs.ub.tu-berlin.de/publizieren/2019/04/fuenf-fragen-an-barbara-budrich/)

- Fünf Fragen an ... Karin Werner vom Verlag transcript (https://blogs. ub.tu-berlin.de/publizieren/2019/05/fuenf-fragen-an-transcript/)

- Fünf Fragen an ... Bianca Matzek vom Verlag Peter Lang (https:// blogs.ub.tu-berlin.de/publizieren/2019/07/fuenf-fragen-an-biancamatzek-vom-verlag-peter-lang/). 